IN PRACTICE

\title{
Audio computer assisted self interview and face to face interview modes in assessing response bias among STD clinic patients
}

\author{
K G Ghanem, H E Hutton, J M Zenilman, R Zimba, E J Erbelding
}

Sex Transm Infect 2005;81:421-425. doi: 10.1136/sti.2004.013193

See end of article for authors' affiliations .................

Correspondence to: Khalil G Ghanem, MD, Johns Hopkins University, Bayview Medical Center, Division of Infectious Diseases, 4940 Eastern Avenue, B3 North, Suite 352, Baltimore, MD 21224, USA; kghanem@ jhmi.edu

Accepted for publication 10 November 2004

\begin{abstract}
Background: Audio computer assisted self interview (ACASI) may minimise social desirability bias in the ascertainment of sensitive behaviours. The aim of this study was to describe the difference in reporting risk behaviour in ACASI compared to a face to face interview (FFI) among public sexually transmitted diseases (STD) clinic attendees.

Study design: Randomly selected patients attending a public STD clinic in Baltimore, Maryland, sequentially took an ACASI formatted risk behaviour assessment followed by an FFI conducted by a single clinician, with both interview modalities surveying sexual and drug use behaviours. Binary responses were compared using the sign test, and categorical responses were compared using the Wilcoxon signed rank test to account for repeated measures.

Results: 671 (52\% men, mean age 30 years, 95\% African American) of 795 clinic attendees screened consented to participate. Subjects affirmed sensitive sexual behaviours such as same sex contact $(p=0.012)$, receptive rectal sexual exposure $(p<0.001)$, orogenital contact $(p<0.001)$, and a greater number of sex partners in the past month $(p<0.001)$ more frequently with ACASI than with an FFI. However, there were no differences in participant responses to questions on use of illicit drugs or needle sharing.

Conclusions: Among STD clinic patients, reporting of sensitive sexual risk behaviours to clinicians was much more susceptible to social desirability bias than was reporting of illegal drug use behaviours. In STD clinics where screening of sexual risk is an essential component of STD prevention, the use of ACASI may be a more reliable assessment method than traditional FFI.
\end{abstract}

S ocial desirability bias is a type of reporting bias that occurs when individuals deny engaging in what are perceived to be socially undesirable behaviours to avoid stigmatisation. ${ }^{1}$ It is usually inferred from differential reporting between two or more interview modes in comparable but separate samples from the same population. ${ }^{2}$ Social desirability bias can have significant consequences on patient care in the clinical setting, and the validity of data gathered in the research setting. ${ }^{3}$ The quality of service delivered in sexually transmitted diseases (STD) clinics may be particularly compromised if such a bias is present to a significant degree. Unbiased measurements of socially sensitive behaviours are necessary to accurately study patterns of STD acquisition and transmission, implement prevention strategies, and assess their effectiveness. ${ }^{4}$

Recently, computer assisted self interviewing (CASI) has been promoted as an interview mode to limit response bias when gathering sensitive information dealing with behaviours perceived to be socially undesirable. ${ }^{5}$ CASI is a computer based technology whereby respondents answer questionnaires in complete privacy without the direct participation of an interviewer. During interviews using CASI methods, respondents answer questions posed in text on the computer screen; in most cases, questions are also posed in audio while respondents listen over headphones (also referred to as audio-CASI or ACASI), thus making it useful even among individuals with limited reading ability. ACASI has been studied in various populations to obtain behavioural data on illicit drug use, ${ }^{6-8}$ HIV risks, ${ }^{9-11}$ and adolescent behaviours. ${ }^{8}$ There are numerous practical advantages to ACASI formatted surveys: consistency in the way questions are asked thus maximising standardisation; limited handling of data forms, thus protecting participant confidentiality; ease in modifying questionnaires to suit a multilingual study setting, ${ }^{8}$ and decreased staff effort related to data entry. There are also limitations to this technology. The use of CASI may reduce the ability to probe for clarification of responses given or elicit responses that require empathy. It may also enable a participant to "surf" through a survey without seriously considering their responses.

ACASI based interviewing may decrease social desirability bias in participant reporting, though there are no gold standards to validate certain types of responses. In general, response rates obtained in ACASI mode are compared to responses given in face to face interview (FFI) from similar representative samples, and differences in reporting are inferred to be the result of social desirability bias. Applying this reasoning, several studies have documented significant differences in response rates and concluded that social desirability bias existed. ${ }^{8}{ }^{13}$

In this study, we compared responses elicited with ACASI to those elicited with a FFI during the same visit in the same participants presenting for care to an inner city public STD clinic. We focused on questions related to sexual behaviours and to illicit drug use. We compared responses between the two modalities to assess social desirability bias in this population.

Abbreviations: ACASI, audio computer assisted self interview; FFI, face to face interview; IDU, injection drug users; STD, sexually transmitted diseases 


\begin{tabular}{|c|c|c|}
\hline \multirow[b]{2}{*}{ Characteristic } & \multicolumn{2}{|c|}{ Respondents $(n=671)$} \\
\hline & Men & Women \\
\hline Number (\%) & 48 & 52 \\
\hline Age (years) mean & 30.9 & 28.8 \\
\hline \multicolumn{3}{|l|}{ Race/ethnicity (\%) } \\
\hline African American & 97 & 93 \\
\hline White & 3 & 4 \\
\hline Other & 0 & 3 \\
\hline \multicolumn{3}{|l|}{ Education (\%) } \\
\hline No formal schooling & 0 & 0.9 \\
\hline 8 th grade or less & 4.0 & 3.4 \\
\hline Some high school & 17.9 & 21.7 \\
\hline High school diploma & 56.4 & 52.7 \\
\hline Some college & 21.6 & 21.3 \\
\hline \multicolumn{3}{|l|}{ Reason for visit (\%) } \\
\hline Contact to known STI & 12 & 15 \\
\hline Symptoms & 59 & 44 \\
\hline Check up & 29 & 41 \\
\hline HIV infected (\%) & 2.5 & 1.0 \\
\hline \multicolumn{3}{|l|}{ STI (\%) } \\
\hline Gonorrhoea & 17 & 6 \\
\hline Chlamydia & 5.0 & 7.2 \\
\hline NGU & 39 & N/A \\
\hline Trichomoniasis & N/A & 11.7 \\
\hline
\end{tabular}

\section{METHODS}

\section{Participants}

All individuals between the ages of 18 years and 65 years presenting to the Baltimore City Health Department Eastern STD Clinic between July 2000, and August 2001 for STD care were eligible and were randomly approached and sequentially recruited to participate in a cross sectional study on the prevalence of mood disorders and their relation to STD risk behaviours.

\section{Measures}

Following informed consent, participants took an ACASI formatted interview including questions on number and types of sex partners, condom use during different types of sexual contact, drug use, and questions from a depressive symptoms screening tool, ${ }^{14}$ all as part of a study with separate aims related to mood and STD risk. This procedure was done in a private room. They then were evaluated by a single female clinician who performed a standardised STD clinic risk assessment in FFI format for the purposes of routine clinical care, along with medical history and STD examination. The clinician was trained on performing the clinical risk assessment to ensure consistency between the two testing modalities, and reproducibility of the FFI mode among all study participants. She was monitored by clinical supervisory staff (EJE) periodically over the course of the study for added quality assurance. Participants had the option of not answering questions in either interview mode. Only responses to questions asked in the FFI that were the same as questions posed in the ACASI formatted risk assessment formed the basis of this analysis.

\section{Human subject considerations}

The study was approved by the institutional review boards of the Johns Hopkins Medical Institutions and the Baltimore City Health Department. Informed consent was obtained from all participants. The consent explicitly stated that study data were protected from outside disclosures by a certificate of confidentiality issued by the Department of Health and Human Services.

\section{Statistical analysis}

All data analyses were performed using STATA (version 8.0, College Station, TX, USA). We compared all participants' individual responses in the ACASI formatted risk assessment to responses in the FFI. Analyses were stratified by age and sex. We used the sign test for comparisons involving binary responses, and the Wilcoxon signed rank test for categorical ones. These statistical techniques were chosen as they took into account the nature of the study design which yielded repeated measures on the same individual (the same individuals being compared by ACASI and FFI modes). p Values of $<0.05$ were considered statistically significant.

\section{RESULTS}

\section{Study participants}

Of 795 patients approached for study participation, 671 (84\%) consented to participate and completed the initial interview sequences and clinical evaluation. Men were more likely to refuse study participation than were women (81 of 401 men refused versus 43 of 394 women; $p=0.001$ ), but those refusing to participate were no different in age or race from those who consented. Table 1 summarises the demographic and clinical characteristics of study participants. More than $50 \%$ of participants had a high school diploma or its equivalent, and $23 \%$ had some college experience. Less than $1 \%$ of participants had no formal schooling

\section{Sexual behaviours}

Table 2 compares the responses to questions about sexual behaviours given in the ACASI as compared to the FFI. Participants were more likely to admit to having multiple sex partners in the past 30 days in the ACASI as compared to the FFI $(p=0.001)$. This response bias remained significant in analyses stratified either by age or by gender. All participants were more likely to admit to oral-genital exposures in the ACASI than the FFI $(p<0.001)$. Women were more likely to report receptive rectal exposures $(p<0.001)$ in the ACASI than the FFI. In the age stratified analysis, a similar trend was observed in all age groups, but this observation was driven mostly by the female respondents. 


\begin{tabular}{|c|c|c|c|}
\hline Question & FFI (\%) & ACASI (\%) & p Value \\
\hline Exchanged sex for money/drugs $(n=661)$ & 10.1 & 13.3 & $<0.05$ \\
\hline Men & 5.8 & 5.8 & NS \\
\hline Women & 14 & 20 & $<0.001$ \\
\hline Age $18-24$ & 1.3 & 6.7 & $<0.001$ \\
\hline Age $25-64$ & 15.2 & 17.1 & NS \\
\hline Sexual orientation (opposite, same, both) $(n=571)$ & $99,0.4,0.6$ & $97,1.4,1.6$ & $<0.05$ \\
\hline No of sex partners in past month $(0-1,2-5,>5)(n=572)$ & $65,34.1,0.9$ & $60.3,36.4,3.3$ & $<0.001$ \\
\hline Men & $49.5,49.1,1.4$ & $45.8,49.1,5.1$ & $<0.05$ \\
\hline Women & $79.7,20,0.3$ & $73.9,24.4,1.7$ & $<0.05$ \\
\hline Age $18-24$ & $67.6,31.9,0.5$ & $61.1,36.1,2.8$ & $<0.05$ \\
\hline Age 25-64 & $63.4,35.5,1.1$ & $59.7,36.6,3.7$ & $<0.05$ \\
\hline Genital exposure $^{*}(n=547)$ & 99.5 & 96.7 & $<0.05$ \\
\hline Men & 99.6 & 95.8 & $<0.05$ \\
\hline Women & 99.3 & 97.5 & NS \\
\hline Age $18-24$ & 99.5 & 96.6 & NS \\
\hline Age 25-64 & 99.4 & 96.8 & $<0.05$ \\
\hline Oral exposure $(n=540)$ & 44.6 & 56.3 & $<0.001$ \\
\hline Men & 41 & 56 & $<0.001$ \\
\hline Women & 47.9 & 56.6 & $<0.001$ \\
\hline Age 18-24 & 42.5 & 54.1 & $<0.001$ \\
\hline Age $25-64$ & 45.6 & 57.6 & $<0.001$ \\
\hline Receptive rectal exposure $(n=291)$ & 6.5 & 20.6 & $<0.001$ \\
\hline Men & 0.3 & 0.7 & NS \\
\hline Women & 6.2 & 19.9 & $<0.001$ \\
\hline Age $18-24$ & 1.7 & 7.2 & $<0.001$ \\
\hline Age 25-64 & 4.8 & 13.1 & $<0.001$ \\
\hline \multicolumn{4}{|c|}{$\begin{array}{l}\text { n, the number of patients who answered the question in both interview modes. } \\
\text { "Genital exposure refers to the insertion of the penis into the vagina; Oral exposure refers to orogenital contact (mouth to penis or mouth to vagina); Rectal } \\
\text { exposure refers to receptive anal intercourse (penis to anus). NS, not significant. The Wilcoxon signed rank test was used to compare categorical responses (sexual } \\
\text { orientation and number of sex partners in the past month), and the sign test was used to compare binary responses. ACASI, audio computer assisted interview; FFI, } \\
\text { face to face interview; IDU, injection drug users. }\end{array}$} \\
\hline
\end{tabular}

Overall, participants were more likely to admit to ever having same sex exposures in the ACASI interview than the FFI $(p=0.012)$; they were also more likely to endorse items related to having ever exchanged sex for money or drugs in the ACASI interview $(p=0.01)$. In analyses stratified by age and gender, women $(\mathrm{p}<0.001)$ and respondents less than 25 years of age $(p<0.001)$ demonstrated a response bias between interview modes for these categories of responses, while men and older respondents did not.

\section{Drug using behaviours}

Table 3 summarises the drug using behaviours measured in our participants. Overall, approximately $10 \%$ of the participants admitted to ever injecting illicit drugs, and there was no difference in reporting illicit injection activity in ACASI compared to FFI. Women were more likely to report injection drug use in FFI than in ACASI; neither men nor different age groups showed this discrepancy although they tended to report more IDU in FFI. There was no significant reporting bias between interview modes among those reporting IDU.

\section{Missing answers}

Participants could opt out of answering questions in either interview mode. For the drug related questions, $<1 \%$ of subjects did not answer the questions in each interview mode. For questions related to sexual behaviour, 16\% of participants taking the ACASI chose not to answer the questions in contrast to $0.3 \%$ in the FFI. Of the 378 patients who skipped the rectal exposure question in ACASI, 377 (99.7\%) denied rectal exposure during the FFI. Similarly, of the 117 patients who skipped the oral exposure question in ACASI, $92(78.6 \%)$ denied oral exposure during the FFI. Of the 98 patients who opted out of reporting the number of sex partners they had in the past 30 days in ACASI, 85 (86.7\%) reported having $0-1$ partner during the FFI.

\section{DISCUSSION}

This study demonstrates a strong social desirability bias in the reporting of sensitive sexual behaviours among STD clinic attendees. We found that study participants were much more likely to endorse certain sensitive sexual behaviours by ACASI than in the FFI, suggesting that in our population,

Table 3 Responses elicited to the drug related questions comparing FFI and ACASI

\begin{tabular}{llcl}
\hline Question & FFI (\%) & ACASI (\%) & p Value \\
\hline IDU ever (n=669) & 10.1 & 9.0 & NS \\
Men & 11 & 11 & NS \\
Women & 9.4 & 7.1 & NS \\
Age 18-24 & 1.2 & 1.2 & NS \\
Age 25-64 & 15.2 & 13.3 & NS \\
Needle sharing* $(n=52)$ & 69.2 & 67.3 & NS \\
Men & 66.7 & 63.3 & NS \\
Women & 72.7 & 72.7 & NS \\
Age 18-24 & 0 & 0 & \\
Age 25-64 & 68 & 66 & \\
\hline
\end{tabular}

*Among participants admitting to injection drug use.

NS, not significant. The sign test was used to compare the binary responses. ACASI, audio computer assisted interview; FFI, face to face interview; IDU, injection drug users. 
social desirability bias operates during the reporting of sensitive sexual behaviours in standard clinical practice. This bias is notable because these patients are presenting for care at an STD clinic, where they should expect to answer questions about their sexual risk behaviours. Substantial social desirability bias of patients in face to face discussions with clinical providers may impact the quality of clinic based STD prevention services in several important ways. In some practice settings, selective screening guidelines for chlamydia diagnostic testing in women are based upon number of recent sex partners reported to the clinician, so that underreporting may lead to undetected chlamydia infection. Also, under-reporting of number of sex partners in any clinic setting will limit disease control measures by compromising the effectiveness of partner notification and referral services. Similarly, incomplete reporting of certain sexual practices may hinder delivery of appropriate clinical care. Patient denial of anal receptive intercourse typically limits clinical specimen collection from this anatomical site thus compromising the effectiveness of diagnostic and therapeutic interventions.

Though male respondents showed no evidence of reporting bias with certain behaviours such as rectal exposure and prostitution, female respondents were more likely to admit to such practices with ACASI compared to FFI. Less than $1 \%$ of male respondents in our study admitted to receptive anal intercourse in either ACASI or FFI. Other published studies reported a significant social desirability bias in behaviours related to same sex contact (including mutual masturbation, oral sex, and anal intercourse), prostitution, and number of sexual partners when comparing interviewer administered questionnaires and ACASI responses. ${ }^{8}$ The low response rates in our study may reflect low participation in same sex contact among males in our clinic, or an equally consistent underreporting of the behaviour in both interview modes.

We found little evidence of bias in comparing our population's reporting of IDU and needle sharing behaviours with ACASI and FFI. Only the subgroup of female participants was more likely to admit to IDU in FFI rather than during the ACASI interview. This finding is in contradistinction to other studies that have shown significantly increased rates of reporting such sensitive drug use beha-

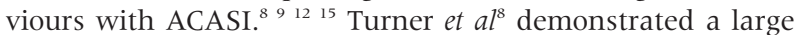
positive response bias favouring reporting of IDU with ACASI. Similarly, Des Jarlais et al found a response bias between ACASI and FFI with more reporting of "sensitive" drug use behaviours in ACASI, and less reporting of "approved" behaviours such as using alcohol wipes to clean before injection. Macalino et $a l^{11}$ found no difference in reporting IDU between CASI and FFI in either HIV negative or positive groups. The cohort in this latter report was recruited from the same inner city population of Baltimore that comprises the patient population attending the STD clinic in this study. Our finding of no response bias for IDU reporting in most subgroups may indicate a high level of understanding of personal HIV risks among these patients and a desire to get STD/HIV testing as a component of clinical services in this setting.

Our study has limitations. As is true with many behavioural studies, there is no validation of the self reported behaviours with biological markers. Our inferences of bias are derived from comparison of the responses given by the same person obtained when varying interview modes. Other studies, however, have included biological variables as a validation of some self reported data. ${ }^{12}$ Thus, some of the differences reported in our study that are attributed to social desirability bias may reflect other inherent biases. Furthermore, in our study, under-reporting on certain stigmatising behaviours (drug use and receptive rectal contact in men) may have occurred with both interview modes which would have made it impossible to detect any response bias. As previously mentioned, specific mode biases detected in special populations, such as urban STD clinic attendees, might not generalise to other groups. Finally, 16\% of respondents opted out of answering questions regarding sexual behaviours in the ACASI group compared to the FFI. Answers given by these participants during the FFI favoured the less stigmatising behaviour. While we believe that the degree of non-response for this question would have made it less likely to detect a response bias even when present, we cannot verify this assumption from our data.

All of our participants first underwent ACASI interview immediately followed by an FFI administered by a single clinician. We do not know whether respondents might have felt pressure to maintain consistency in their responses with the sequential risk assessments. Because the sequence always began with the ACASI, any reporting bias for consistency's sake would have biased our results towards the null. It is not possible, however, to rule out an order effect bias in this study. Some individuals may have felt that they could avoid answering sensitive questions in the FFI because they had already provided the answer earlier with ACASI. A randomised crossover design changing interview sequences would have allowed us to address this, but the flow scheme we used was designed to address our main study aim related to mood and risk behaviour. The sex of the clinician may also have influenced some of the differential responses observed between the male and female participants. More research is needed to ascertain how differential reporting, often attributed to social desirability bias, may differ by clinical provider and by patient.

Finally, the fact that a single research clinician was responsible for the clinical risk assessment meant greater standardisation of the FFI, but makes our results less generalisable to reporting biases that might exist in data gathered in STD clinics as a whole. Future studies evaluating the role of ACASI in the STD clinic setting should include multiple interviewers to better reflect standard clinical practice. We have shown that social desirability bias exists for STD clinic patients when reporting certain sensitive sexual behaviours to their clinician. More complete disclosure of these behaviours may improve with confidential interviewing using computer technology, though gender and age may have a role in optimising data collection for sensitive risk behaviours. Further research to evaluate the feasibility of integrating confidential computer interviewing into STD clinic operations, as well as its impact on quality of care, is needed.

\section{ACKNOWLEDGEMENTS}

The authors thank Charlene Wylie for recruitment efforts and the staff of the Baltimore City Health Department Eastern Health District Clinic for their cooperation.

\section{CONTRIBUTORS}

KGG, data collection, data analysis, drafting of manuscript; EJE, study design, data collection, data analysis, drafting of manuscript; $\mathrm{HH}$, data collection, significant revisions to manuscript.; JZ, data analysis, significant revisions to manuscript; RZ, data analysis, significant revisions to manuscript.

\section{Authors' affiliations}

K G Ghanem, H E Hutton, J M Zenilman, E J Erbelding, Johns Hopkins University School of Medicine, Baltimore, MD, USA

R Zimba, New York City Department of Health and Mental Hygiene, New York, NY, USA

E J Erbelding, Baltimore City Health Department, Baltimore, MD, USA

Financial support: NIH R01 MH60066-01A1 (EJE), NIH K24 A101663 (JMZ) and Association of Teachers for Preventive Medicine (KGG). 
Portions of this paper were presented in poster format at the International Society for Sexually Transmitted Diseases Research (ISSTDR), Ottawa, Canada, July 2003.

Conflict of interest: None of the authors report any conflicts of interest.

Human subject considerations: The study was approved by the institutional review boards of the Johns Hopkins Medical Institutions and the Baltimore City Health Department. Informed consent was obtained from all participants. The consent explicitly stated that study data were protected from outside disclosures by a Certificate of Confidentiality issued by the Department of Health and Human Services.

\section{REFERENCES}

1 Rosenthal R, Persinger GW, Fode KL. Experimenter bias, anxiety, and social desirability. Percept Mot Skills 1962;15:73-4.

2 Newman JC, Des J, Turner CF, et al. The differential effects of face-to-face and computer interview modes. Am J Public Health 2002;92:294-7.

3 King M, Bruner G. Social desirability bias: a neglected aspect of validity testing. Psychology and Marketing 2003;17:79-103.

4 Gregson S, Zhuwau T, Ndlovu J, et al. Methods to reduce social desirability bias in sex surveys in low-development settings: experience in Zimbabwe. Sex Transm Dis 2002;29:568-75.

5 O'Reilly J, Hubbard M, Lessler P, et al. Audio and video computer-assisted self-interviewing: preliminary tests of new technologies for data collection. J Off Stat 1994; 10:197-214.

6 Williams ML, Freeman RC, Bowen AM, et al. A comparison of the reliability of self-reported drug use and sexual behaviors using computer-assisted versus face-to-face interviewing. AIDS Educ Prev 2000;12:199-213.
7 Bangsberg DR, Bronstone A, Chesney MA, et al. Computer-assisted selfinterviewing (CASI) to improve provider assessment of adherence in routine clinical practice. J Acquir Immune Defic Syndr. 2002;31: S107-11, S107S111, (Suppl 3).

8 Turner CF, Ku L, Rogers SM, et al. Adolescent sexual behavior, drug use, and violence: increased reporting with computer survey technology. Science 1998;280:867-73.

9 Des J, Paone D, Milliken J, et al. Audio-computer interviewing to measure risk behaviour for HIV among injecting drug users: a quasi-randomised trial. Lancet 1999;353:1657-61.

10 Van Griensven F, Supawitkul S, Kilmarx PH, et al. Rapid assessment of sexual behavior, drug use, human immunodeficiency virus, and sexually transmitted diseases in northern thai youth using audio-computer-assisted selfinterviewing and noninvasive specimen collection. Pediatrics 2001;108:E13.

11 Macalino GE, Celentano DD, Latkin C, et al. Risk behaviors by audio computer-assisted self-interviews among HIV-seropositive and HIVseronegative injection drug users. AIDS Educ Prev 2002; 14:367-78.

12 Murphy DA, Durako S, Muenz LR, et al. Marijuana use among HIV-positive and high-risk adolescents: a comparison of self-report through audio computer-assisted self-administered interviewing and urinalysis. Am J Epidemiol 2000;152:805-13.

13 Gribble JN, Miller HG, Cooley PC, et al. The impact of T-ACASI interviewing on reported drug use among men who have sex with men. Subst Use Misuse 2000;35:869-90.

14 National Institute on Drug Abuse. Risk behavior assessment. Rockville, MD: National Institute on Drug Abuse, 2003.

15 Metzger DS, Koblin B, Turner C, et al. Randomized controlled trial of audio computer-assisted self-interviewing utility and acceptability in longitudinal studies. HIVNET Vaccine Preparedness Study Protocol Team. Am J Epidemiol 2000;152:99-106. 